\title{
Mining New Plipastatins and Increasing the Total Yield Using CRISPR/Cas9 in Genome-modified Bacillus subtilis 1A751
}

Dandan ZOU ${ }^{a}$, Sarah Wanjiku Maina ${ }^{a}$, Fengmin Zhang ${ }^{b}$, Zhenzhen YANa, Liping $\mathrm{DING}^{\mathrm{a}}$, Yuting SHAO ${ }^{\mathrm{a}}$, Zhihong XIN $\mathrm{X}^{\mathrm{a}, *}$

a Key Laboratory of Food Processing and Quality Control, College of Food Science and Technology, Nanjing Agricultural University, Nanjing 210095, P.R. China

b Testing Center, Yangzhou University, Wenhui East Rd. 48, Yangzhou 225009, China

*Corresponding author at: Key Laboratory of Food Processing and Quality Control, College of Food Science and Technology,

Nanjing Agricultural University, Nanjing 210095, Jiangsu Province, P.R. China

Tel./fax: +862584395618.

E-mail address: xzhfood@njau.edu.cn (Zhihong Xin). 


\section{SUPPLEMENTAL TABLES and FIGURES}

Table S1. Strains and plasmids used in this study.

Table S2. Primers used in this study.

Table S3. The predicted functions of the pps gene cluster.

Table S4. Analysis sgRNA target sequences online of different websites and its knockout efficiency in strains.

Figure S1. Bioinformatic analysis of plipastatin gene cluster and its structures. The plipastatin operon (pps, $38 \mathrm{~kb}$ ) consists of five genes: ppsABCDE, which catalyze 10 amino acids into plipastatin. $\mathrm{R}$ stands for $\beta$-hydroxy fatty acid ( $\beta$-OH FA) side chain with 15-21 carbon atoms.

Figure S2. Workflow for the construction of gene knockout plasmid. Three steps for assembling the $\operatorname{srf} A B-A C$ gene delete plasmid: Step 1 was performed by Golden Gate to insert 20 nt sgRNA between the BsaI sites; then, the left and right homology arms were amplified by PCR from genomic DNA (gDNA). The Order Gene Assemble method (OGAB) was used in step 3 to integrate homology arms into SfiI sites.

Figure S3. LC-ESI-MS spectrometry analysis of fermentation products of 1A751-pps-srf $\triangle \operatorname{srfAB}-A C$. LC-ESI-MS spectrum displays mass ions ranging from $m / z 1400$ to 1600 .

Figure S4. GC-MS analysis of TMS derivatives of fatty acid side chains. (a) The TIC spectrum of the retention time for goal compounds was 15-43 min. Ion fragments of TMS derivatives of $\mathrm{C}_{16}(\mathbf{b}), \mathrm{C}_{17}(\mathbf{c}), \mathrm{C}_{18}(\mathbf{d}), \mathrm{C}_{19}(\mathbf{e}), \mathrm{C}_{20}(\mathbf{f})$, and $\mathrm{C}_{21}$ (g) saturated $\beta-\mathrm{OH}$ fatty acid chains, respectively. 
Table S1 Strains and plasmids used in this study.

\begin{tabular}{|c|c|c|}
\hline strains or plasmids & characteristics & source \\
\hline \multicolumn{3}{|l|}{ strains } \\
\hline B. subtilis $1 \mathrm{~A} 751$ & his nprR2 nprE18 DaprA3 DeglS102 DbglTbglSRV & laboratory stock \\
\hline B. subtilis 168 & $\operatorname{trp} C 2$, pheA1 & laboratory stock \\
\hline B. subtilis 1A751 $4 p p s$ & his nprR2 nprE18 DaprA3 DeglS102 & laboratory constructed \\
\hline & $\begin{array}{l}\text { DbglTbglSRV } \triangle p p s ; \text { engineered host strain for } \\
\text { herterologous expression }\end{array}$ & \\
\hline B. subtilis & B. subtilis $1 \mathrm{~A} 751$ contained TAR captured & $\left(\mathrm{Hu}, \mathrm{Nan}\right.$ et al. 2018) ${ }^{l}$ \\
\hline $\begin{array}{c}1 \mathrm{~A} 751 \Delta p p s+p p s+ \\
s f p+\operatorname{deg} Q\end{array}$ & plipastatin biosynthetic gene cluster; $\mathrm{Spec}^{\mathrm{R}}$ & \\
\hline B. subtilis & B. subtilis 1A751 $\Delta p p s+p p s+s f p+\operatorname{deg} Q$ & this study \\
\hline $\begin{array}{c}1 \mathrm{~A} 751 \Delta p p s+p p s+ \\
s f p+\operatorname{deg} Q+ \\
\Delta s r f A B-A C\end{array}$ & $\begin{array}{l}\text { derivate: deleted part of surfactin biosynthetic gene } \\
\text { cluster; } \operatorname{Spec}^{\mathrm{R}}\end{array}$ & \\
\hline E. coli $\mathrm{DH} 5 \alpha$ & 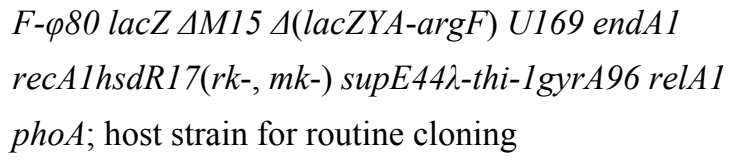 & $\begin{array}{l}\text { Vazyme Biotech Co., } \\
\text { Ltd }\end{array}$ \\
\hline \multicolumn{3}{|l|}{ plasmids } \\
\hline pCAPB2+PPS & $\begin{array}{l}\mathrm{Amp}^{\mathrm{R}}, \mathrm{Spec}^{\mathrm{R}}, \operatorname{trp} 1 ; \mathrm{pCAPB} 2-\mathrm{HASD} \text { derivate, gene } \\
\text { cluster expression vector, containing plipastatin } \\
\text { biosynthetic gene cluster }\end{array}$ & $\left(\mathrm{Li}, \mathrm{Li}\right.$ et al. 2015) ${ }^{2}$ \\
\hline pJOE8999 & $\begin{array}{l}\text { Kana }^{\mathrm{R}} \text {; a shuttle vector containing pUCori } \\
\text { promoter, the temperature-sensitive replicon, } \\
\text { mannose-inducible promoter } \operatorname{PmanP} \text {, cas } 9 \text { and } \\
g R N A\end{array}$ & (Altenbuchner 2016) $^{3}$ \\
\hline pJOE8999+ sgRNA 1 & $\begin{array}{l}\text { Kana }^{\text {R }} \text {; pJOE } 8999 \text { containing } 2 \mathrm{~kb} \text { homology } \\
\text { template and sgRNA } 1\end{array}$ & this study \\
\hline pJOE8999+ sgRNA 2 & $\begin{array}{l}\text { Kana }^{\mathrm{R}} \text {; pJOE } 8999 \text { containing } 2 \mathrm{~kb} \text { homology } \\
\text { template and sgRNA } 2\end{array}$ & this study \\
\hline pJOE8999+ sgRNA 3 & $\begin{array}{l}\text { Kana }^{\mathrm{R}} \text {; pJOE } 8999 \text { containing } 2 \mathrm{~kb} \text { homology } \\
\text { template and sgRNA } 3\end{array}$ & this study \\
\hline
\end{tabular}


Table S2. Primers used in this study.

\begin{tabular}{|c|c|c|}
\hline primers & $\left(5^{\prime}-3^{\prime}\right)$ sequence & $\begin{array}{l}\text { restriction } \\
\text { sites }\end{array}$ \\
\hline sgRNA 1-F & TACGttcaaactccttctcattgt & BsaI \\
\hline sgRNA 1-R & AAACacaatgagaaggagtttgaa & BsaI \\
\hline sgRNA 2-F & TACGcgttgtatgtgacagccggg & BsaI \\
\hline sgRNA 2-R & AAACcceggctgtcacatacaacg & BsaI \\
\hline sgRNA 3-F & TACGaatgaaagaatggacaaagg & BsaI \\
\hline sgRNA 3-R & AAACcctttgtccattctttcatt & BsaI \\
\hline P1 & $\begin{array}{l}\text { AAGGCCAACGAGGCCGCCTCATGTACA } \\
\text { AAACCG }\end{array}$ & SfiI \\
\hline $\mathrm{P} 2$ & $\begin{array}{l}\text { AAGGCCATGTTGGCCGAAGCAAAGCTG } \\
\text { CGCGAA }\end{array}$ & SfiI \\
\hline $\mathrm{P} 3$ & $\begin{array}{l}\text { AAGGCCAACATGGCCTCATGTACACGTC } \\
\text { CGGCA }\end{array}$ & SfiI \\
\hline P4 & $\begin{array}{l}\text { AAGGCCTTATTGGCCCGTCGTTAAAGGA } \\
\text { AGCTC }\end{array}$ & SfiI \\
\hline P5 & CTGCGGAATAGTCGCATT & - \\
\hline P6 & AAACACAATGAGAAGGAGTTTGAA & - \\
\hline P7 & CTGCGGAATAGTCGCATT & - \\
\hline P8 & GAAGCAAAGCTGCGCGAA & - \\
\hline P9 & GTACCCCCCTATGTTTTC & - \\
\hline $\mathrm{P} 10$ & AGCTGGCACGACAGGTTT & - \\
\hline P11 & GCTACAACATGCCATCTG & - \\
\hline P12 & TCCCTGATGAGCCTCATA & - \\
\hline P13 & GAAATGAGCTGGCTCTGC & - \\
\hline P14 & GCATGATCTGCCGGATTC & - \\
\hline
\end{tabular}

Note: Restriction sites are marked in bold; 20 bp protospacer sequences are represented in lowercase letters.

no

restriction

sites. 
Table S3. The predicted functions of the pps gene cluster.

Proteins encoded by the pps gene cluster and open reading frames adjacent to the $p p s$ cluster as well as their proposed function and size.

\begin{tabular}{|c|c|c|c|c|}
\hline$\Rightarrow$ other genes & - & NRPS genes & & 48.57-kb \\
\hline protein & $\begin{array}{l}\text { size } \\
\text { (aa) }\end{array}$ & proposed function & $\begin{array}{c}\text { identity/ } \\
\text { similarity } \\
(\%)\end{array}$ & accession No. \\
\hline YoeA & 463 & $\begin{array}{l}\text { Putative efflux transporter } \\
\text { [Bacillus velezensis] }\end{array}$ & $100 / 100$ & AEW31016.1 \\
\hline YoeX & 325 & $\begin{array}{l}\text { Putative epimerase } \\
\text { [Bacillus velezensis] }\end{array}$ & $100 / 100$ & AEW31017.1 \\
\hline $\mathrm{DacC}$ & 501 & $\begin{array}{l}\text { Penicillin-binding protein } \\
\text { [Bacillus velezensis }]\end{array}$ & $100 / 100$ & AEW31018.1 \\
\hline PpsA & 2452 & $\begin{array}{l}\text { Plipastatin synthetase [Bacillus } \\
\text { velezensis] }\end{array}$ & $97 / 100$ & AEW31019.1 \\
\hline PpsB & 2548 & $\begin{array}{l}\text { Plipastatin synthetase [Bacillus } \\
\text { velezensis] }\end{array}$ & $98 / 100$ & AEW31020.1 \\
\hline $\mathrm{PpsC}$ & 2545 & $\begin{array}{l}\text { Plipastatin synthetase [Bacillus } \\
\text { velezensis] }\end{array}$ & $98 / 100$ & AEW31021.1 \\
\hline PpsD & 3591 & $\begin{array}{l}\text { Plipastatin synthetase [Bacillus } \\
\text { velezensis] }\end{array}$ & $98 / 100$ & AEW31022.1 \\
\hline PpsE & 1272 & $\begin{array}{l}\text { Plipastatin synthetase [Bacillus } \\
\text { velezensis] }\end{array}$ & $98 / 100$ & AEW31015.1 \\
\hline YngL & 130 & $\begin{array}{l}\text { Integral inner membrane protein } \\
\text { [Bacillus velezensis] }\end{array}$ & $97 / 100$ & AEW31023.1 \\
\hline YngK & 510 & $\begin{array}{l}\text { Hypothetical protein } \\
\text { [Bacillus velezensis] }\end{array}$ & $100 / 100$ & AEW31024.1 \\
\hline YngJ & 390 & $\begin{array}{l}\text { Acyl-CoA dehydrogenase } \\
\text { [Bacillus velezensis] }\end{array}$ & $96 / 100$ & AEW31025.1 \\
\hline YngI & 549 & $\begin{array}{l}\text { Short-chain acyl-CoA } \\
\text { synthetase }[\text { Bacillus velezensis }]\end{array}$ & $100 / 100$ & AEW31026.1 \\
\hline YngH & 444 & $\begin{array}{l}\text { Acetyl-CoA carboxylase biotin } \\
\text { carboxylase subunit [Bacillus } \\
\text { velezensis] }\end{array}$ & $100 / 100$ & AEW31027.1 \\
\hline
\end{tabular}


Table S4. Analysis sgRNA target sequences online of different websites and its knockout efficiency in strains.

\begin{tabular}{|c|c|c|c|c|c|c|c|}
\hline \multirow{2}{*}{$\begin{array}{l}\text { target } \\
\text { gene }\end{array}$} & \multirow{2}{*}{$\begin{array}{l}\text { RGEN target } \\
\left(5^{\prime} \text { 'to } 3 \text { ') }\right.\end{array}$} & \multirow{2}{*}{ PAM } & \multirow{2}{*}{ strand } & \multirow{2}{*}{$\begin{array}{l}\text { out of } \\
\text { frame } \\
\text { score }\end{array}$} & \multicolumn{2}{|c|}{$\begin{array}{l}\text { gene editing } \\
\text { efficiency } / \%\end{array}$} & \multirow{2}{*}{ design Web } \\
\hline & & & & & B.S 168 & HYM & \\
\hline \multirow{3}{*}{$\operatorname{SrfAB}-A C$} & $\begin{array}{l}\text { ACAATGAGAA } \\
\text { GGAGTTTGAA }\end{array}$ & AGG & - & 81.7 & 90 & 41.67 & $\begin{array}{l}\text { Crispr RGEN } \\
\text { Tools }{ }^{4}\end{array}$ \\
\hline & $\begin{array}{c}\text { CCCGGCTGTCA } \\
\text { CATACAACG }\end{array}$ & CGG & + & 78.5 & 11.11 & 11.11 & Benchling ${ }^{5}$ \\
\hline & $\begin{array}{l}\text { AATGAAAGAA } \\
\text { TGGACAAAGG }\end{array}$ & GGG & + & 71.0 & 70 & 0 & CRISPOROR ${ }^{6}$ \\
\hline
\end{tabular}

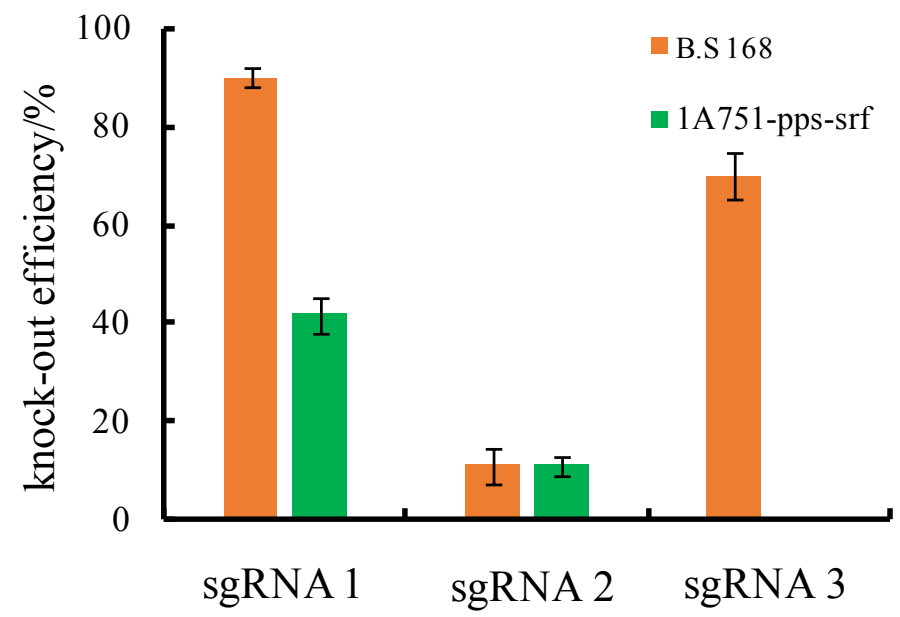




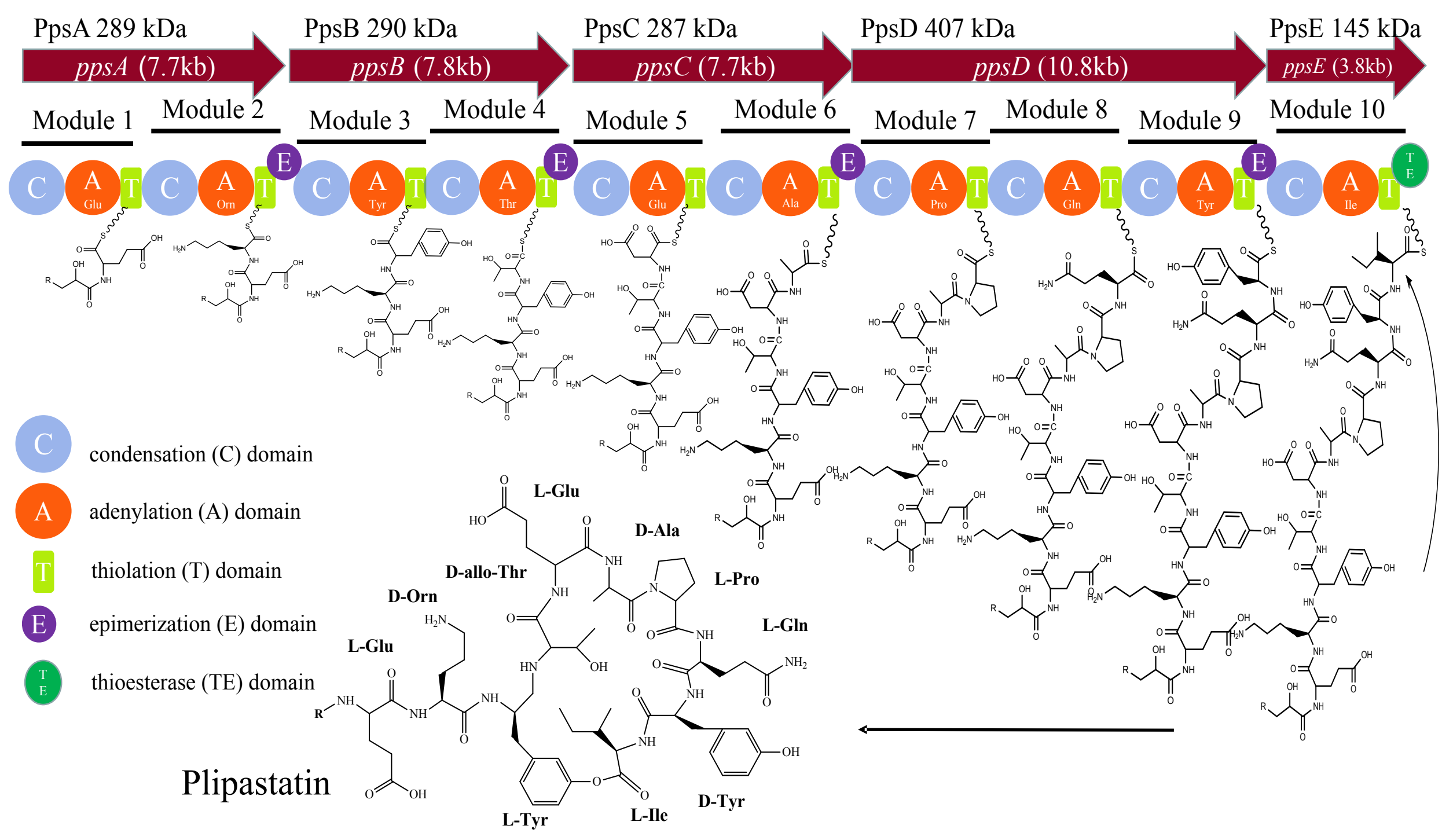


$\mathrm{R}=\mathrm{C} 15-\mathrm{C} 21 \beta-\mathrm{OHFA}$<smiles>CCCCCCCCCCCCC(O)CC(C)=O</smiles><smiles></smiles><smiles>CCCCCCCCCCCCCCCCC(O)CC(C)=O</smiles><smiles>CC(=O)CC(O)CCCCCCCCCCCCCCCCl</smiles><smiles>CC(=O)CCCCCCCCCCCCCCCCC(O)CC(C)=O</smiles><smiles>CCCCCCCCCCCCCCCCCCCC(O)CC(C)=O</smiles>

Figure S1. Bioinformatic analysis of plipastatin gene cluster and its structures. The plipastatin operon (pps, $38 \mathrm{~kb}$ ) consists of five genes: ppsABCDE, which catalyze 10 amino acids into plipastatin. $\mathrm{R}$ stands for $\beta$-hydroxy fatty acid ( $\beta$-OH FA) side chain with 15-21 carbon atoms. 


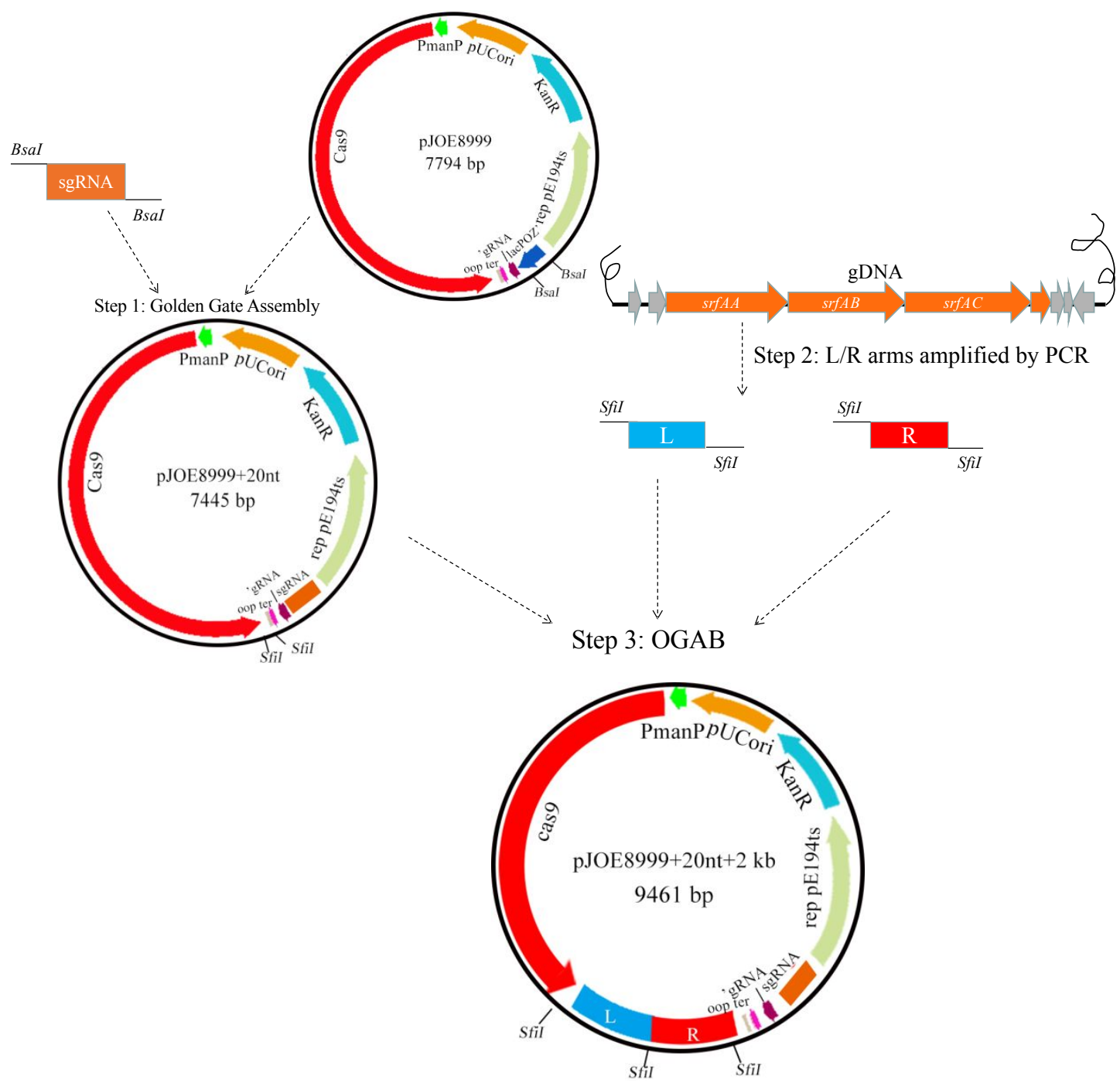

Figure S2. Workflow for the construction of gene knockout plasmid. Three steps for assembling the $\operatorname{srf} A B-A C$ gene delete plasmid: Step 1 was performed by Golden Gate to insert $20 \mathrm{nt} \operatorname{sgRNA}$ between the BsaI sites; then, the left and right homology arms were amplified by PCR from genomic DNA (gDNA). The Order Gene Assemble method (OGAB) was used in step 3 to integrate homology arms into SfiI sites. 
20191205-ZDD 1093 (8.039) Cm (1063:1097)

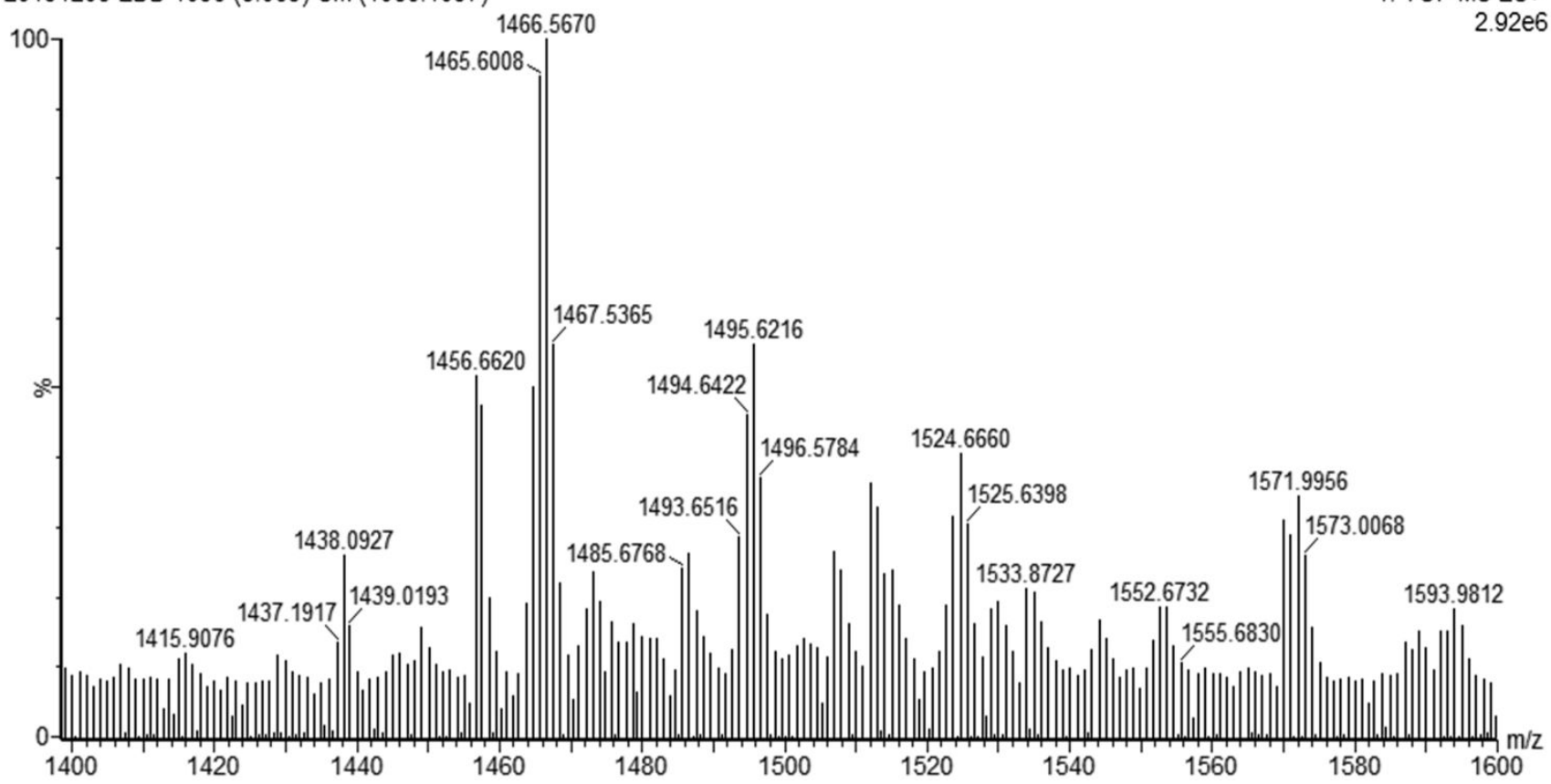

Figure S3. LC-ESI-MS spectrometry analysis of fermentation products of 1A751-pps-srf $\triangle s r f A B-A C$. LC-ESI-MS spectrum displays mass ions ranging from $m / z 1400$ to 1600 . 
a.

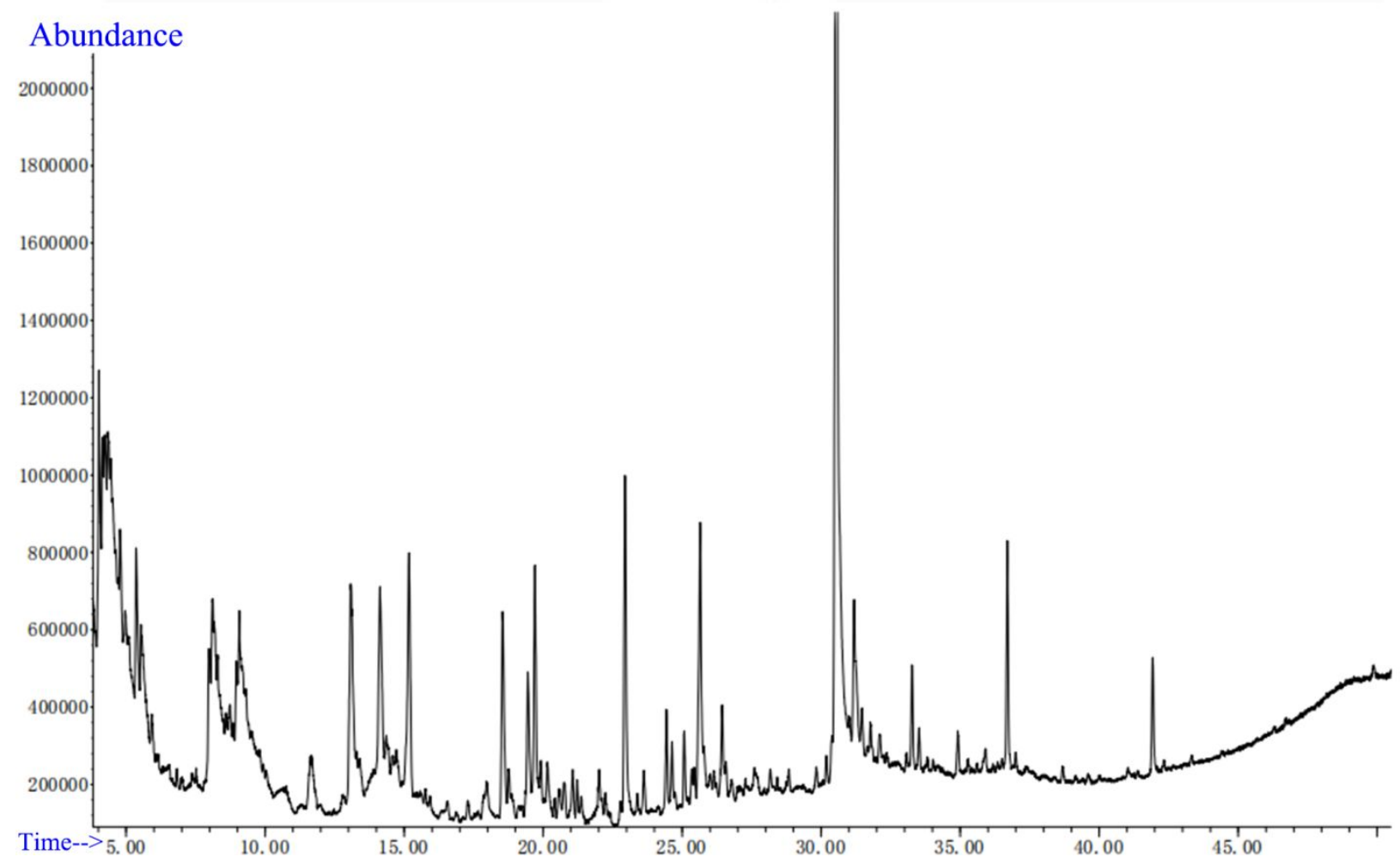

b.

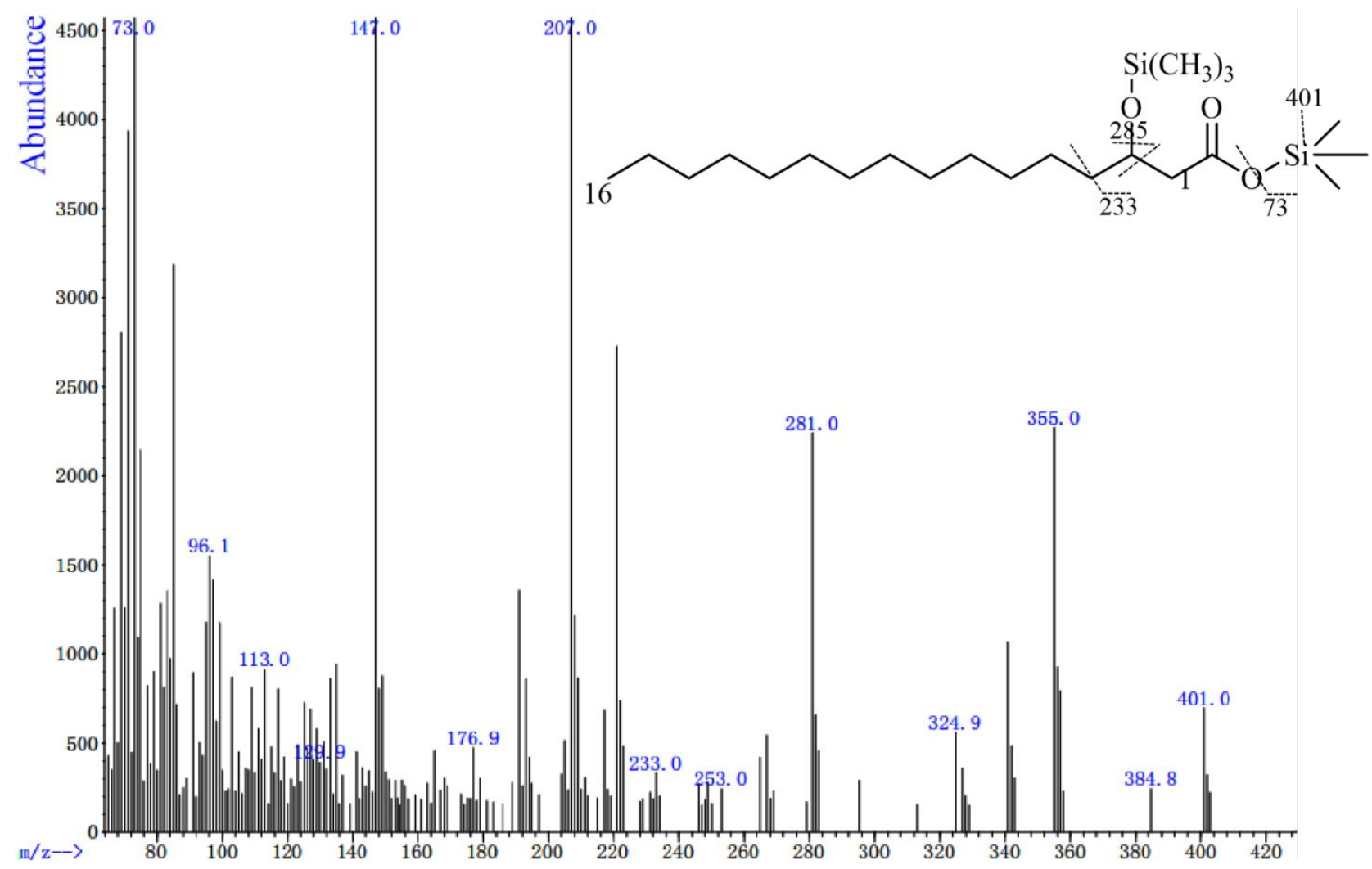

c. 


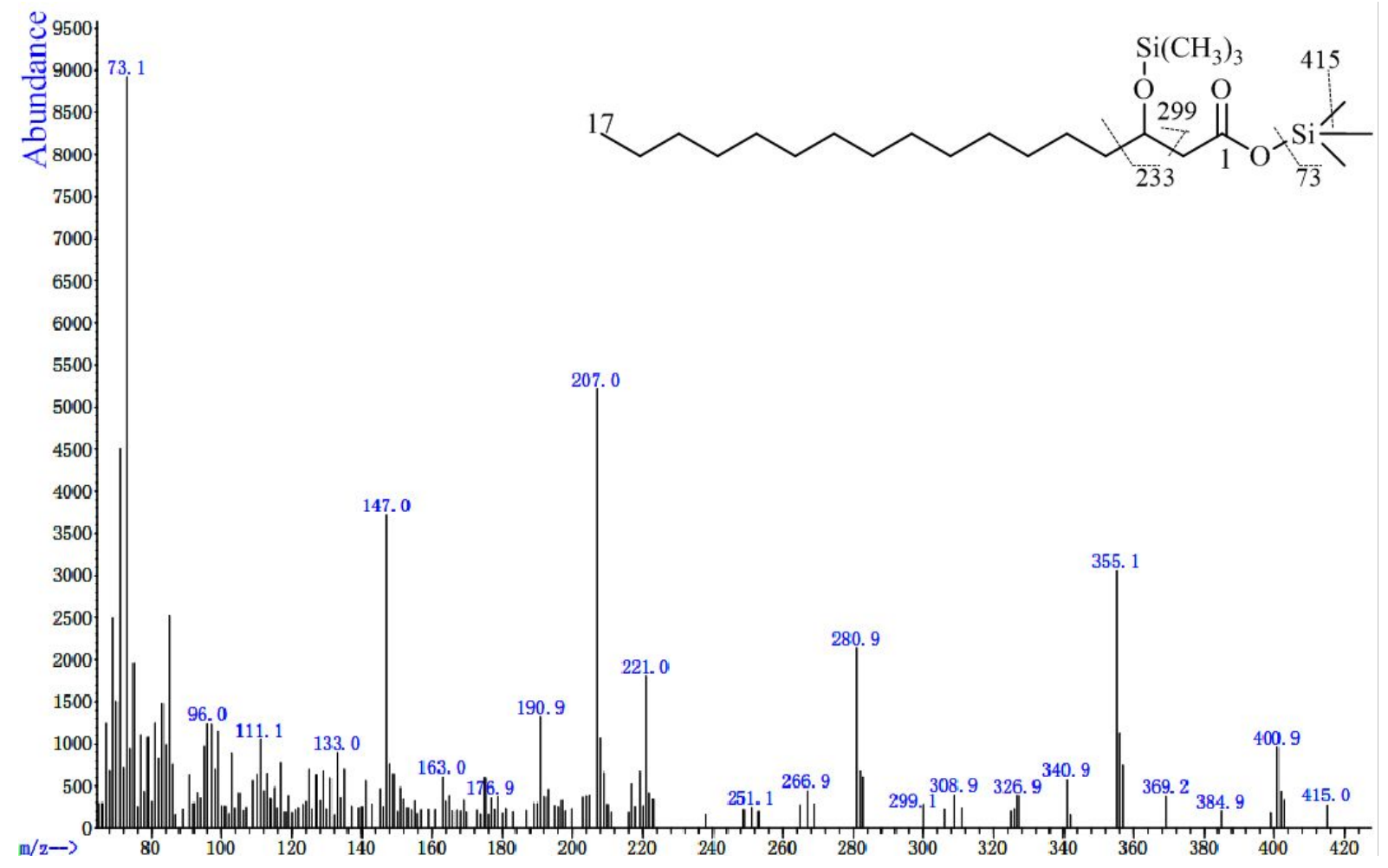

d.

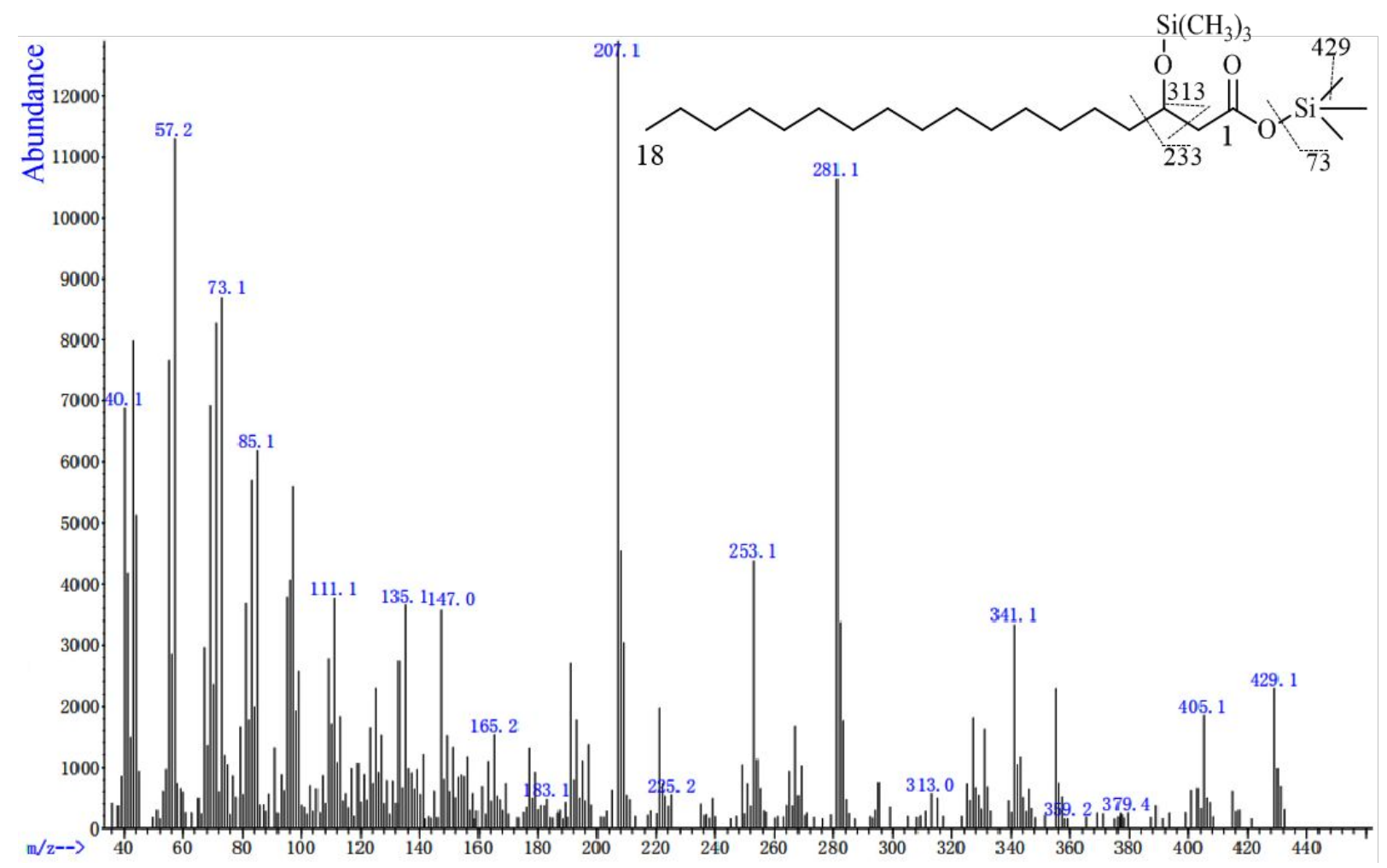

e. 


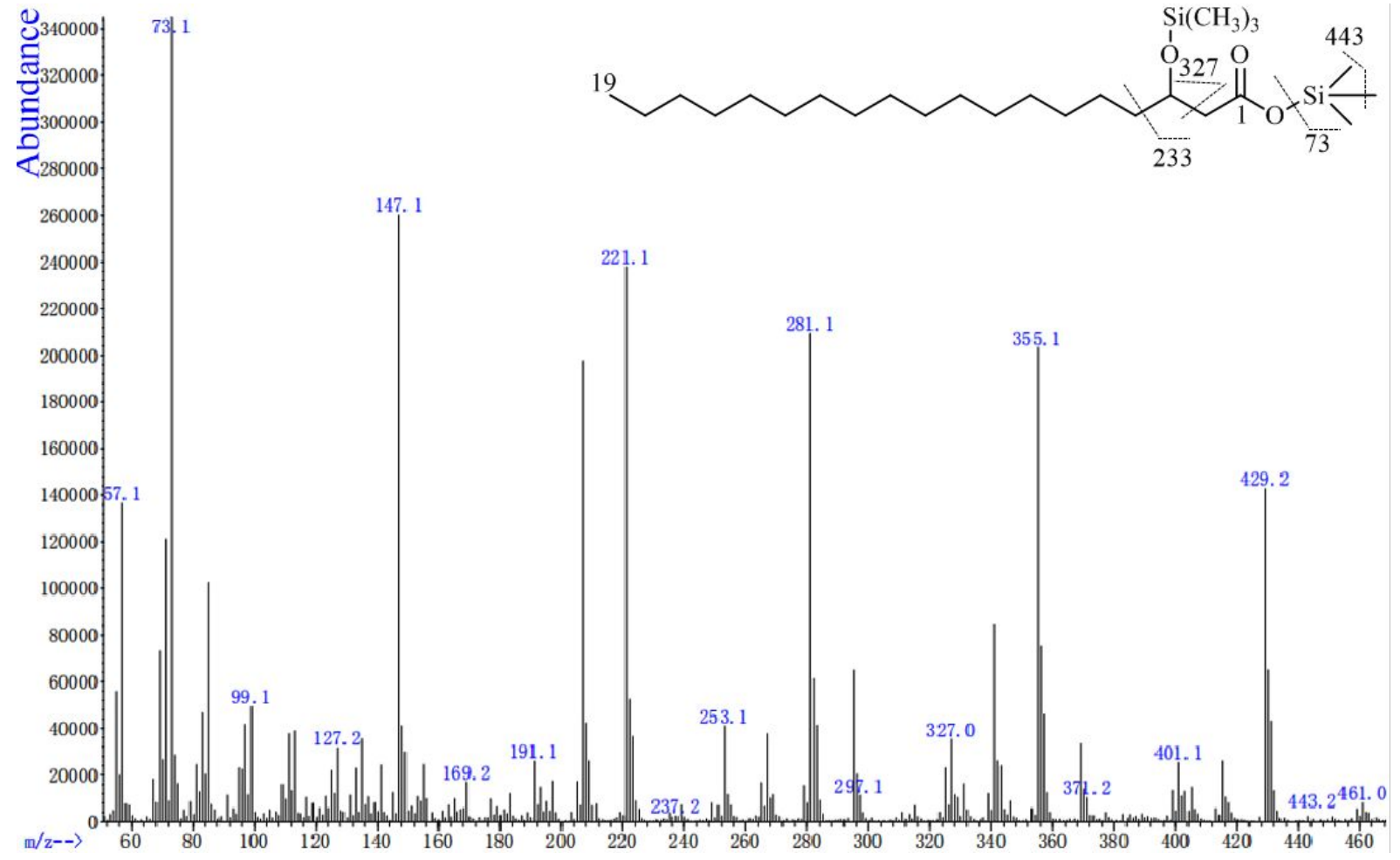

f.

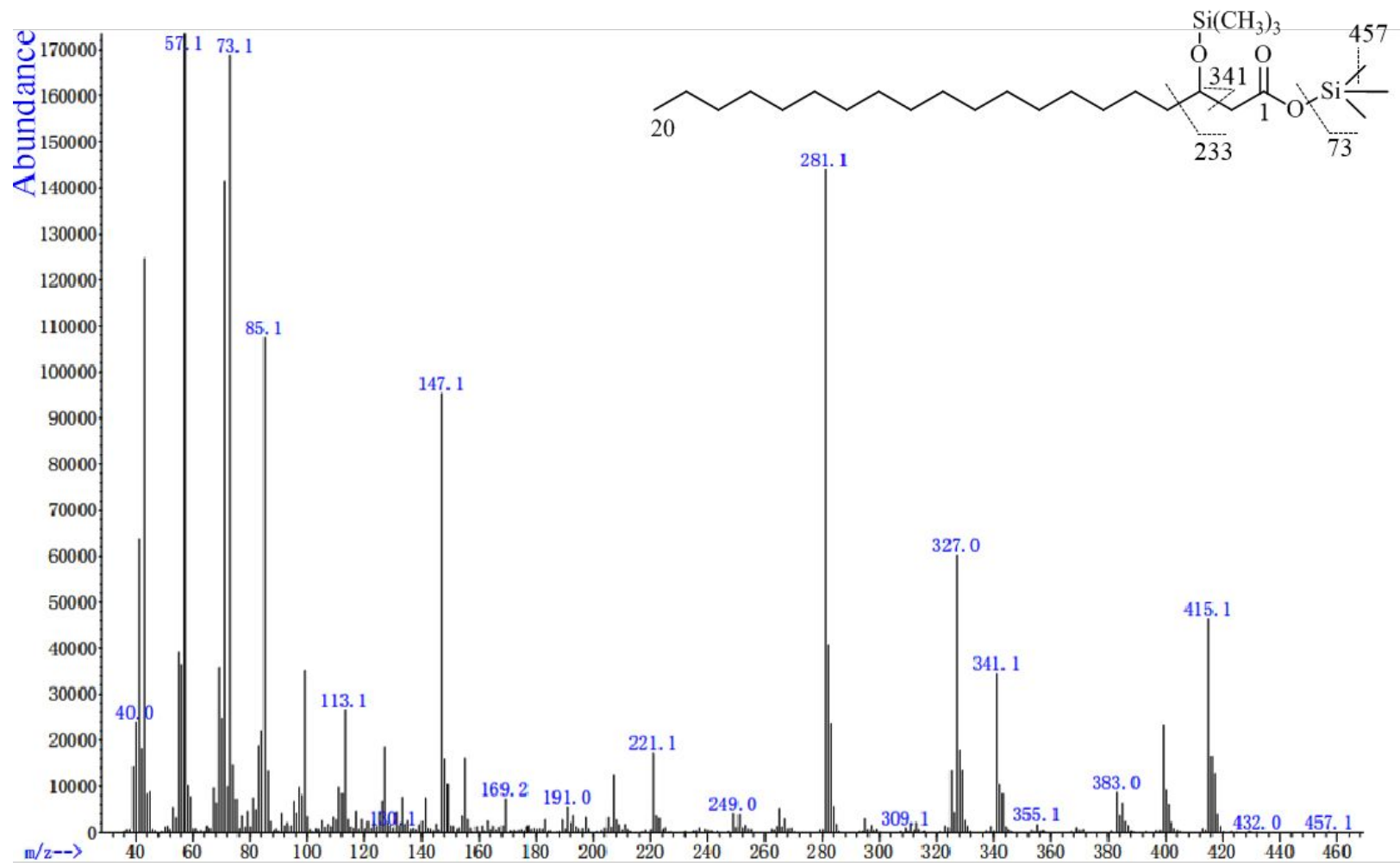

g. 


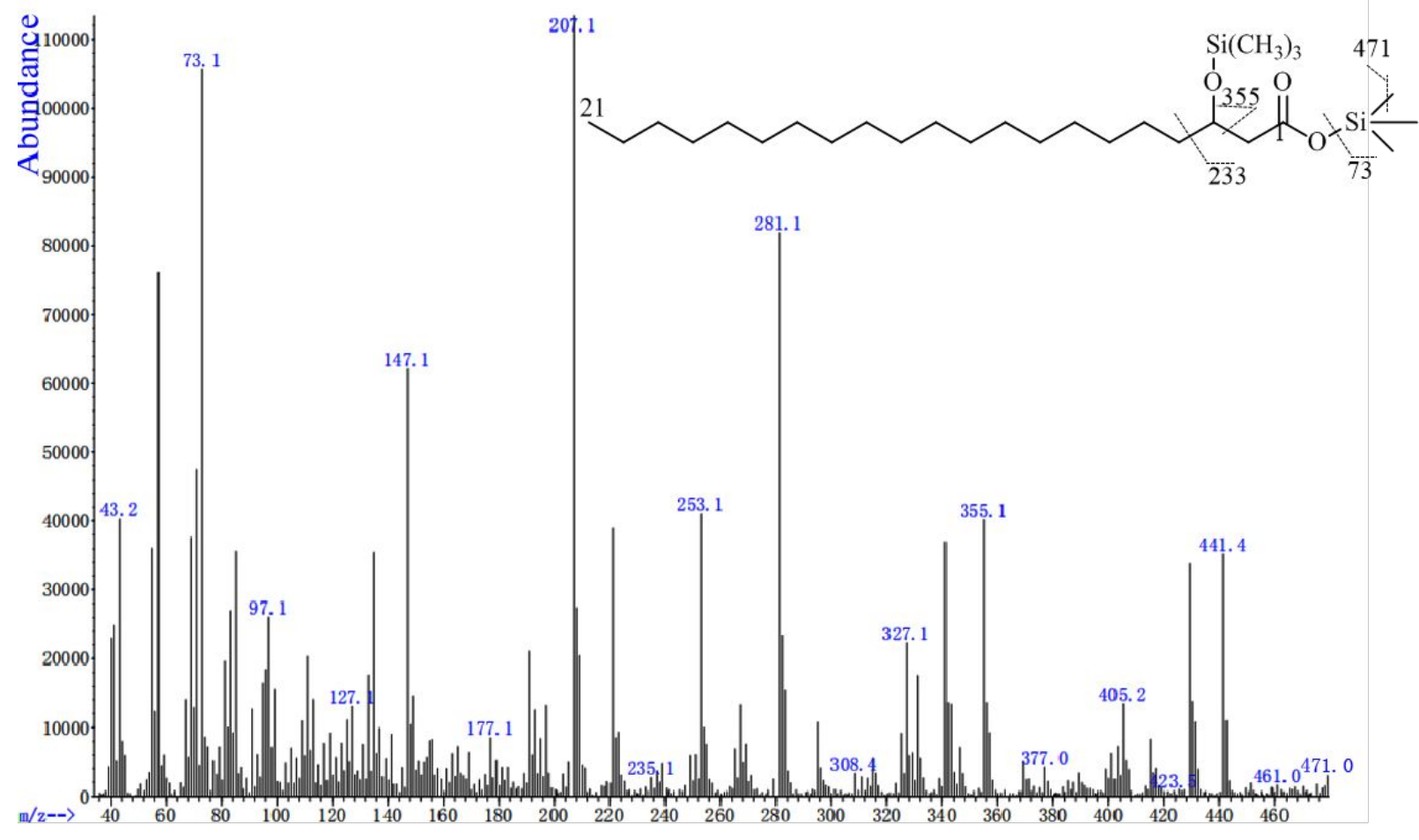

Figure S4. GC-MS analysis of TMS derivatives of fatty acid side chains. (a) The TIC spectrum of the retention time for goal compounds was 15-43 min. Ion fragments of TMS derivatives of $\mathrm{C}_{16}(\mathbf{b}), \mathrm{C}_{17}(\mathbf{c}), \mathrm{C}_{18}(\mathbf{d}), \mathrm{C}_{19}(\mathbf{e}), \mathrm{C}_{20}(\mathbf{f})$, and $\mathrm{C}_{21}(\mathbf{g})$ saturated $\beta-\mathrm{OH}$ fatty acid chains, respectively.

\section{References}

(1) Hu, Y. M.; Nan, F.; Maina, S. W.; Guo, J.; Wu, S. L.; Xin, Z. H., Clone of Plipastatin Biosynthetic Gene Cluster by Transformation-associated Recombination Technique and High Efficient Expression in Model Organism Bacillus subtilis. J. Biotechnol. 2018, 288, 1-8.

(2) Li, Y. X.; Li, Z. R.; Yamanaka, K.; Xu, Y.; Zhang, W. P.; Vlamakis, H.; Kolter, R.; Moore, B. S.; Qian, P. Y., Directed Natural Product Biosynthesis Gene Cluster Capture and Expression in the Model Bacterium Bacillus subtilis. Scientific Reports 
2015, 5,7 .

(3) Altenbuchner, J., Editing of the Bacillus subtilis Genome by the CRISPR-Cas9 System. Appl Environ Microbiol 2016, 82, 5421-5427.

(4) Zhou, C.; Liu, H.; Yuan, F.; Chai, H.; Wang, H.; Liu, F.; Li, Y.; Zhang, H.; Lu, F., Development and Application of a CRISPR/Cas9 Aystem for Bacillus licheniformis Genome Editing. Int J Biol Macromol 2019, 122, 329-337.

(5) Haeussler, M.; Schonig, K.; Eckert, H.; Eschstruth, A.; Mianne, J.; Renaud, J. B.; Schneider-Maunoury, S.; Shkumatava, A.; Teboul, L.; Kent, J.; Joly, J. S.; Concordet, J. P., Evaluation of Off-target and On-target Scoring Algorithms and Integration into the Guide RNA Selection Tool CRISPOR. Genome Biol. 2016, 17, 12.

(6) Uniyal, A. P.; Mansotra, K.; Yadav, S. K.; Kumar, V., An Overview of Designing and Selection of sgRNAs for Precise Genome Editing by the CRISPR-Cas9 System in Plants. 3 Biotech 2019, 9, 19. 